\title{
Stroke mimic diagnoses presenting to a hyperacute stroke unit
}

\author{
Authors: Ang Dawson, ${ }^{A}$ Geoffrey $C$ Cloud, ${ }^{B}$ Anthony $C$ Pereira ${ }^{C}$ and Barry $]_{\text {Moynihan }}{ }^{B}$
}

Stroke services have been centralised in several countries in recent years. Diagnosing acute stroke is challenging and a high proportion of patients admitted to stroke units are diagnosed as a non-stroke condition (stroke mimics). This study aims to describe the stroke mimic patient group, including their impact on stroke services. We analysed routine clinical data from 2,305 consecutive admissions to a stroke unit at St George's Hospital, London. Mimic groupings were derived from 335 individual codes into 17 groupings. From 2,305 admissions, 555 stroke mimic diagnoses were identified $(24.2 \%)$ and $72 \%$ of stroke mimics had at least one stroke risk factor. Common mimic diagnoses were headache, seizure and syncope. Medically unexplained symptoms and decompensation of underlying conditions were also common. Median length of stay was 1 day; a diagnosis of dementia $(p=0.028)$ or needing MRI $(p=0.006)$ was associated with a longer stay. Despite emergency department assessment by specialist clinicians and computed tomography brain, one in four suspected stroke patients admitted to hospital had a nonstroke diagnosis. Stroke mimics represent a heterogeneous patient group with significant impacts on stroke services. Co-location of stroke and acute neurology services may offer advantages where service reorganisation is being considered.

KEYWORDS: Diagnosis, mimic, MRI, patient journey, risk factors, service, stroke, stroke mimic

\section{Introduction}

A wide range of diagnoses may present like stroke, also called stroke mimics. ${ }^{1,2}$ We previously reported that stroke mimics comprise a quarter of direct admissions to the hyperacute stroke unit (HASU) at St George's Hospital, London ${ }^{3}$ and similar rates have been reported internationally. ${ }^{4,5}$ Diagnosis of stroke mimics may be a consequence of an acute stroke care

Authors: ${ }^{\text {A }}$ specialist registrar in neurology, St. George's University Hospitals NHS Foundation Trust, London, UK; ${ }^{\mathrm{B}}$ Consultant stroke physician, St. George's University Hospitals NHS Foundation Trust, London, UK; C Consultant neurologist, St. George's University Hospitals NHS Foundation Trust, London, UK pathway and become a significant component of acute stroke services. Magnetic resonance imaging (MRI) is often important in diagnosing stroke and mimics. ${ }^{6,7}$ We report our single-centre experience of this patient group and their impact on acute stroke services in terms of length of stay (LOS) and imaging resources within the NHS.

\section{Methods}

We reviewed all patients admitted to St George's Hospital HASU from 1 October 2011 to 31 December 2012. The unit serves a population of 1.6 million people. Suspected stroke cases are seen by the neurology team in the emergency department (ED). After initial ED triage, which includes computed tomography (CT) brain, patients are directly admitted to the HASU for further evaluation and treatment within a 4-hour government target window. Those diagnosed as stroke mimics are treated, discharged or referred on as appropriate. A list of patients admitted to the HASU with a non-stroke diagnosis was retrospectively identified from the prospectively collected national database, Stroke Improvement National Audit Programme (SINAP). ${ }^{8}$ The electronic discharge summaries, test results, clinic letters and radiology reports for each patient were reviewed. Accurate data available for each patient were age, gender, length of hospital stay from admission to the HASU until discharge from the hospital, presence or absence of dementia or psychiatric condition, discharge from the HASU itself or referral on. Data were available on stroke risk factors: hypertension, diabetes, atrial fibrillation, ischaemic heart disease, smoking, hypercholesterolaemia and cardiac failure. Previous stroke or transient ischaemic attack (TIA) were also available but analysed separately to avoid double counting. While these risk factors are not strictly relevant for stroke mimics, we hypothesised that the presence of stroke risk factors prompted HASU admission for further assessment.

Ethical approval of the SINAP audit and associated data linkage was granted by the Ethics and Confidentiality Committee of the National Information Governance Board.

\section{Diagnostic categorisation}

ICD-10 codes for each individual primary diagnoses were available. There were 335 individual codes (S1 - ICD-10 codes). Therefore, similar ICD-10 code diagnoses were amalgamated (eg patients with migraine and other headache disorders 
Table 1. Diagnostic categories of stroke mimics

\begin{tabular}{|c|c|c|c|c|c|c|c|c|c|}
\hline Category & $\mathbf{N}$ & $\begin{array}{l}\text { Mean age, } \\
\text { years (SD) }\end{array}$ & $\begin{array}{l}\text { Median LOS, } \\
\text { days (IQR) }\end{array}$ & Female, n (\%) & tPA & RFs & MRI & $\begin{array}{l}\text { Previous } \\
\text { stroke/TIA }\end{array}$ & $\begin{array}{l}\text { Home directly } \\
\text { from HASU }\end{array}$ \\
\hline Headache \& migraine aura ${ }^{+}$ & 85 & $49.3(16.1)$ & $1(0-1)$ & $62(73)$ & 1 & $56 \%$ & $67 \%$ & $12 \%$ & $99 \%$ \\
\hline Seizure & 66 & $66.7(18.3)$ & $2(1-4)$ & $30(45)$ & 3 & $76 \%$ & $41 \%$ & $44 \%$ & $70 \%$ \\
\hline Unexplained & 60 & $59.3(20.0)$ & $1(1-2)$ & $40(67)$ & 2 & $72 \%$ & $65 \%$ & $18 \%$ & $93 \%$ \\
\hline Decompensation & 44 & $67.3(17.0)$ & $2(1-3)$ & $25(57)$ & 1 & $95 \%$ & $55 \%$ & $84 \%$ & $57 \%$ \\
\hline Presyncope \& syncope & 39 & $73.2(15.8)$ & $2(1-2)$ & $25(38)$ & & $85 \%$ & $38 \%$ & $33 \%$ & $87 \%$ \\
\hline Vestibular & 38 & $66.2(13.7)$ & $1(1-2)$ & $18(47)$ & & $82 \%$ & $74 \%$ & $8 \%$ & $92 \%$ \\
\hline Psychiatric & 36 & $53.8(19.4)$ & $2(1-2)$ & $21(58)$ & 6 & $69 \%$ & $61 \%$ & $17 \%$ & $86 \%$ \\
\hline Neurology & 35 & $60.6(22.3)$ & $2(1.0-4.5)$ & $16(46)$ & 1 & $57 \%$ & $71 \%$ & $14 \%$ & $66 \%$ \\
\hline Mononeuropathy & 33 & $54.9(16.3)$ & $1(1-1)$ & $15(45)$ & 1 & $55 \%$ & $52 \%$ & $9 \%$ & $100 \%$ \\
\hline Infection & 21 & $80.9(13.1)$ & $3(2-5)$ & $14(67)$ & & $81 \%$ & $48 \%$ & $43 \%$ & $48 \%$ \\
\hline Neuromuscular \& pain & 21 & $56.0(17.2)$ & $1(1-2)$ & $10(48)$ & & $67 \%$ & $67 \%$ & $10 \%$ & $81 \%$ \\
\hline Medical & 18 & $73.8(17.8)$ & $1.5(1-3)$ & $11(61)$ & & $89 \%$ & $56 \%$ & $22 \%$ & $78 \%$ \\
\hline Malignant & 15 & $73.3(12.5)$ & $4(2.0-7.5)$ & $6(40)$ & & $73 \%$ & $87 \%$ & $20 \%$ & $67 \%$ \\
\hline Falls & 14 & $80.7(10.7)$ & $2(3.0-3.75)$ & $5(36)$ & & $86 \%$ & $57 \%$ & $43 \%$ & $36 \%$ \\
\hline Confusion & 12 & $75.8(10.7)$ & $2(1.0-3.5)$ & $6(50)$ & & $83 \%$ & $67 \%$ & $50 \%$ & $58 \%$ \\
\hline Drug \& alcohol toxicity & 12 & $60.6(19.6)$ & $1.5(1-3)$ & $6(50)$ & & $50 \%$ & $33 \%$ & $25 \%$ & $75 \%$ \\
\hline Trauma & 6 & $72.5(19.4)$ & $2.5(2-3)$ & $3(50)$ & & $50 \%$ & $50 \%$ & $0 \%$ & $50 \%$ \\
\hline
\end{tabular}

${ }^{\dagger}$ Migraine and other primary headache disorders, including acephalgic migraine aura.

$\mathrm{HASU}=$ hyperacute stroke unit; IQR = interquartile range; $\mathrm{LOS}=$ length of stay; $\mathrm{MRI}=$ magnetic resonance imaging; $\mathrm{N}=$ number of patients; RFs = patients with at least 1 stroke risk factor; TIA = transient ischaemic attack; tPA = patients administered thrombolysis.

had 10 different ICD-10 codes and were merged into the 'headache and migraine aura' grouping). Further merging of diagnostic groups yielded 17 groups with usable statistical data (Table 1); eight were primarily neurological categories - headache and migraine aura, mononeuropathy (including those with Bell's palsy, radial nerve palsy or oculomotor or abducens nerve palsies), neurology (diagnoses affecting the central nervous system, eg demyelination), neuromuscular and pain (mainly radiculopathy or polyneuropathy), psychiatric (mainly functional illness), seizures, traumatic brain injury and vestibular disorders; nine were conditions that would normally be managed by medical teams and included confusion (eg delirium), decompensation (of an underlying medical or neurological condition, usually stroke), drug and alcohol toxicity, falls, infection (eg pneumonia or urinary tract infection), malignant disease, medical conditions, presyncope/ syncope and unexplained conditions. Unexplained conditions were typically presumed functional syndromes without positive diagnostic evidence.

Descriptive statistics are provided for theses 17 categories. Comparisons using interval data were analysed using Analysis of Variance (ANOVA). Comparisons using ordinal or nominal data were analysed using the Kruskal-Wallis oneway analysis of variance by ranks, Wilcoxon signed ranks test or chi-squared test.

To calculate the effect of MRI on bed occupancy, we looked at only those patients who went directly home $(n=442)$. Their mean LOS was 1.694 days (Total bednights $=749(442 \times 1.694))$. MRI added an average of 1.6 days to the LOS. We subtracted
1.6 from the LOS for each of these patients with a threshold of 0 (to avoid negative LOS). The mean LOS for those patients was reduced to 0.67 days (total bednights $296 / 442$ patients). For the HASU, the total bednight count was 9,140 for the time period of the study. Therefore, if MRI was not a factor in delaying discharge and was applied to all patients, occupancy would have been reduced from $8.2 \%$ to $3.2 \%$ ( $749 / 9,140$ to $296 / 9,140)$.

\section{Results}

During the study period, there were 2,305 consecutive admissions to the HASU and 555 were stroke mimics $(24.1 \%$ of the whole cohort over 15 months). $56.5 \%$ had primarily neurological causes. The mean age of the stroke mimics was 62.8 years (SD 19.3); $55 \%$ were female; $72 \%$ had at least one of the previously mentioned risk factors for stroke; $27 \%$ had had a previous stroke or TIA; $58 \%$ had an MRI scan.

In each category, $50 \%$ or more patients had at least one risk factor for stroke. Overall, a significantly greater proportion of patients had risk factors for stroke $(\mathrm{p}<0.0001$, Wilcoxon signed ranks). Similarly, patients were overall significantly more likely to undergo MRI scanning ( $\mathrm{p}=0.045$, Wilcoxon signed ranks).

Headache and migraine aura and seizures comprised the largest mimic groups. Patients with unexplained symptoms or decompensation of a previous medical or neurological condition were the next commonest groups. There was no significant difference in the gender mix of patients in any of the categories except in headache and migraine aura, where the majority of 


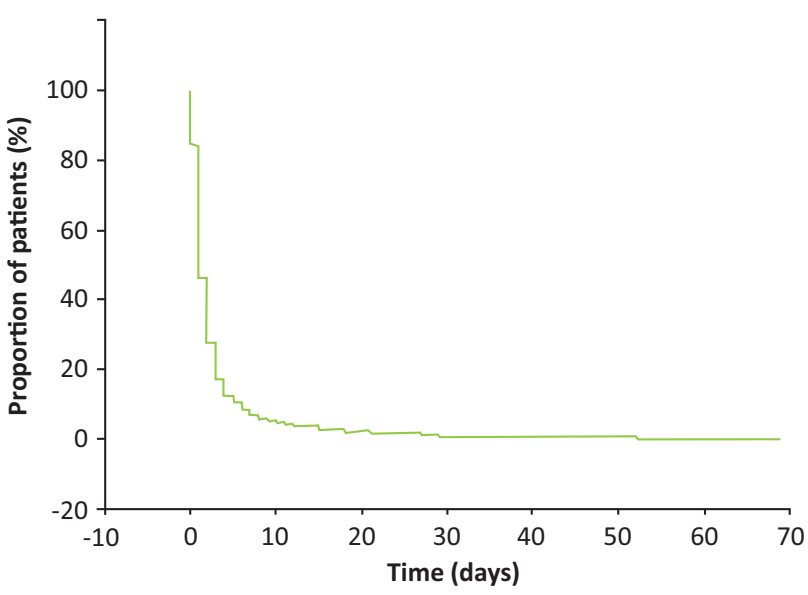

Fig 1. Proportion of stroke mimic patients remaining in hospital following admission.

patients were female. Fifteen patients with a final stroke mimic diagnosis were thrombolysed; most commonly those with psychiatric conditions or seizures. There were no adverse events related to administering thrombolysis in this group.

Stroke mimic patients were discharged rapidly from the HASU (Fig 1). The mean LOS was 2.8 (SD 5.7) days. The median LOS was 1 (IQR 1-3) day with $16.2 \%$ of the stroke mimic patients being discharged home the same day. A further $38 \%$ were discharged the next day; $6.7 \%$ remained longer than 1 week and $3.4 \%$ longer than a fortnight.

LOS in the whole cohort was not affected by age, presence of a psychiatric condition, presence of a previous stroke/TIA or presence of stroke risk factors. Patients stayed significantly longer if they had dementia (4.75 (SD 9.1) days, $\mathrm{p}=0.028$ ANOVA) or awaiting MRI (3.5 (SD 7.2) days versus 1.9 (SD 2.5) days $\mathrm{p}=0.0006$ ANOVA). LOS was similar in the various diagnostic categories. Only patients with malignant disease, infections or falls stayed longer but statistical analysis was not performed here given the potential for arbitrary bias in the categories.

Patients who had a previous stroke or TIA were more prevalent in the group who were admitted with decompensation of an underlying neurological or medical condition (chisquared test 3.8 (95\% CI 3.1-4.6), $\mathrm{p}<0.0001)$. Prior stroke or TIA was not a significant contributor alone to the other diagnostic categories.

442 patients ( $79.6 \%$ of all mimics) were discharged directly from the HASU (mean LOS 1.69 (SD 2.3) days) while 113 patients (19.4\% of all mimics) were referred on to other hospital teams or other hospitals (mean LOS 7.3 (SD 10.7) days). Patients who had had a fall or infection were more likely to be referred on. Patients not discharged directly from the HASU were significantly older 74.5 (SD 7.46) years versus 62.3 (SD 9.59), $\mathrm{p}=0.00019)$, more likely to have had a prior stroke or TIA ( $40.7 \%$ versus $23.5 \%, \mathrm{p}=0.0002)$, dementia ( $13.3 \%$ versus $5.7 \%, \mathrm{p}=0.005)$ and stroke risk factors $(79.6 \%$ versus $69.9 \%$, $\mathrm{p}=0.04)$.

The 442 (79.6\%) patients who were discharged directly from the HASU spent a total of 749 days in the HASU $(8.2 \%$ of HASU occupancy). The 113 (19.6\%) who were referred on spent 826 days in hospital, equivalent to $9 \%$ of the HASU bed capacity. Therefore, mimics contributed $24.2 \%$ of admissions and between 8.2 to $17.2 \%$ of the HASU bed occupancy.

\section{Discussion}

Accurate diagnosis of acute stroke is not only important in order to select patients for time-dependent reperfusion therapies, such as thrombolysis and thrombectomy, but also to inform appropriate secondary stroke prevention management. Moreover, the diagnosis of stroke has a significant impact on a range of issues such as vocation, driving and insurance premiums for individuals. ${ }^{9,10}$ The drive for early diagnosis of stroke through organised stroke care has created an important cohort of patients with non-stroke diagnoses (stroke mimics). Diagnosis of mimics is arguably as important as the diagnosis of stroke. Our report confirms the wide range of neurological and medical conditions that present as mimic syndromes to stroke services, broadly split between neurological and medical causes.

The HASU appears to be an efficient model for discharging stroke mimics home (median LOS 1 day). There is a high rate of MRI use (57\%) and bed use (8.0-17.2\% of the HASU capacity) in the admitted mimic population. One alternate explanation is the HASU model may paradoxically increase admission in a group of patients who have stroke risk factors for an MRI scan to exclude stroke. This may explain why patients with stroke risk factors or unexplained symptoms or decompensation of a previous medical or neurological condition were prevalent groups. The presence of stroke risk factors per se should not contribute to the diagnosis of a mimic condition. Acute MRI with diffusion weighted imaging sequences is more accurate at diagnosing ischaemic stroke than plain CT, especially in the posterior fossa and in suspected lacunar stroke syndromes. ${ }^{11}$ It is also clinically useful in the context of patients presenting with pre-existing cerebrovascular disease in the context of a suspected seizure, which may or may not be provoked by an acute cerebral infarct. ${ }^{12}$ MRI can identify asymptomatic infarcts as well as evidence of prior intracranial haemorrhage, which can inform stroke aetiological diagnosis and stroke secondary prevention treatment strategies. ${ }^{13,14}$ Similarly, contrast-enhanced MRI is invaluable in differentiating low density change on CT scan from change associated with acute inflammation and tumour.

In each mimic category, $50 \%$ of patients (or more) had at least one risk factor for stroke. While we didn't have a control group, the presence of risk factors may influence the likelihood of being admitted to HASU for further evaluation. $57 \%$ of patients underwent MRI scanning. Having MRI significantly increased the LOS by an average of 1.6 days. If MRI was immediately available and was the main delaying factor, HASU bed occupancy by stroke mimic patients could be potentially reduced from $8.2 \%$ to $3.2 \%$. This calculation illustrates the importance of MRI access for diagnosis and management of mimics, including managing LOS of highcost stroke beds. MRI was reported to not be cost effective in secondary prevention strategies of stroke and TIA but may be very useful for diagnostic purposes and identification of mimics. ${ }^{15}$

Our study has limitations. We only studied the mimics that were admitted to the HASU. A proportion of patients were screened out in the ED. The impact of this patient group 
remains unstudied. The retrospective nature of our study precludes decision analysis of the reasons for admission to HASU. Nevertheless, the high prevalence of stroke risk factors suggests this played a role in prioritising admission and all patients had neurological assessment.

\section{Conclusions}

Despite ED assessment by specialist clinicians and CT brain, one in four suspected stroke patients admitted to hospital have a non-stroke diagnosis, ie acute stroke diagnosis is not always easy. The presence of stroke risk factors may influence the decision to admit. There is a wide range of stroke mimic diagnoses but most are neurological or medical. MRI is often required for diagnosis so better access to emergency MRI may reduce need for admission and reduce LOS. The availability of an acute neurology team is important to diagnose and manage neurological mimic conditions and co-location of stoke and neurology services may be the optimal service configuration, although the evidence is for stroke units to be geographically discrete areas of clinical care. ${ }^{16}$ The stroke mimic patient pathway needs to be included in delivering and improving acute stroke services.

\section{Conflicts of interest}

The authors have no competing interests to declare.

\section{Author contributions}

All authors contributed to the design, analysis of the results and write up. AD extracted the data. BJM and ACP qualified data queries and perform statistical analysis.

\section{References}

1 Lioutas V-A, Sonni S, Caplan LR. Diagnosis and misdiagnosis of cerebrovascular disease. Curr Treat Options Cardiovasc Med 2013;15:276-87.

2 Merino JG, Luby M, Benson RT et al. Predictors of acute stroke mimics in 8187 patients referred to a stroke service. J Stroke Cerebrovasc Dis 2013;22:e397-403.

3 Moynihan B, Davis D, Pereira A et al. Delivering regional thrombolysis via a hub-and-spoke model. J R Soc Med 2010;103:363-9.

4 Karli ski M, Gluszkiewicz M, Członkowska A. The accuracy of prehospital diagnosis of acute cerebrovascular accidents: an observational study. Arch Med Sci 2015;11:530-5.
5 Dupre CM, Libman R, Dupre SI et al. Stroke chameleons. J Stroke Cerebrovasc Dis 2014;23:374-8.

6 Jauch E, Saver J, Adams HPJr et al. Guidelines for the early management of patients with acute ischemic stroke: a guideline for healthcare professionals from the American Heart Association/ American Stroke Association. Stroke 2013;44:870-947.

7 Goyal M, Hoff B, Williams J et al. Streamlined Hyperacute Magnetic Resonance Imaging Protocol Identifies Tissue-Type Plasminogen Activator-Eligible Stroke Patients When Clinical Impression Is Stroke Mimic. Stroke 2016;47:1012-7.

8 SINAP. The Stroke Improvement National Audit Programme. London: RCP, 2012. Available online at www.rcplondon.ac.uk/ projects/stroke-improvement-national-audit-programme-sinap [Accessed 9 June 2016].

9 Daniel K, Wolfe CD, Busch MA, McKevitt C. What are the social consequences of stroke for working-aged adults? A systematic review. Stroke 2009;40:e431-40.

10 Aufman EL, Bland MD, Barco PP, Carr DB, Lang CE. Predictors of return to driving after stroke. Am J Phys Med Rehabil 2013;92:627-34.

11 Chalela JA, Kidwell CS, Nentwich LM et al. Magnetic resonance imaging and computed tomography in emergency assessment of patients with suspected acute stroke: a prospective comparison. Lancet 2007;369:293-8.

12 Kanazawa Y, Morioka T, Arakawa S et al. Nonconvulsive partial status epilepticus mimicking recurrent infarction revealed by diffusion-weighted and arterial spin labeling perfusion magnetic resonance images. J Stroke Cerebrovasc Dis 2015;24:731-8.

13 Baradaran H, Gialdini G, Mtui E et al. Silent brain infarction in patients with asymptomatic carotid artery atherosclerotic disease. Stroke 2016;47:1368-70.

14 Charidimou A, Jaunmuktane $\mathrm{Z}$ et al White matter perivascular spaces: an MRI marker in pathology-proven cerebral amyloid angiopathy? Neurology 2014;82:57-62.

15 Wardlaw J, Brazzelli M, Miranda $\mathrm{H}$ et al. An assessment of the costeffectiveness of magnetic resonance, including diffusion-weighted imaging, in patients with transient ischaemic attack and minor stroke: a systematic review, meta-analysis and economic evaluation. Health Technol Assess 2014;18:1-368, v-vi.

16 Stroke Unit Trialists' Collaboration. Organised inpatient (stroke unit) care for stroke. Cochrane Database Syst Rev 2013;(9):CD000197.

\footnotetext{
Address for correspondence: Dr A Pereira, Department of Neurology, Atkinson Morley Wing, St. George's University Hospitals NHS Foundation Trust, Blackshaw Road, Tooting, London SW17 0QT, UK.

Email: anthony.pereira@stgeorges.nhs.uk
} 